\title{
Evolución del paisaje amazónico desde el Precámbrico
}

\author{
Luís Enrique Gainette Prates ${ }^{1}$
}

\begin{abstract}
Resumen Este ensayo es un estudio acerca de la evolución del paisaje amazónico desde el Precámbrico hasta el presente. Resulta de un intenso levantamiento bibliográfico de la literatura publicada en libros y ensayos científicos nacionales e internacionales. Presenta las evidencias geológicas y climáticas de los cambios en la Amazonia, así como sus principales implicaciones sobre la biodiversidad actual; destacando el punto de vista de los diversos autores sobre las hipótesis acerca del Paleo-amazonas y la formación de la actual cuenca amazónica. El trabajo expone que los procesos geológicos y climáticos fueron determinantes para los distintos y múltiples cambios del paisaje a través del tiempo que afectaron la topografía, el suelo, las formaciones vegetales, y la fauna de Suramérica y de la Amazonia. Hubo un gran impacto de estos eventos en la formación de los reductos forestales y no-forestales que contribuyeron con la especiación y divergencia de especies que están presentes en los diferentes hábitats de la Amazonia actual. Hoy, el desagüé del Amazonas, en el Atlántico, aporta intensa sedimentación que es transportada por las mareas, formando los extensos manglares del norte de Brasil. Sin embargo, los efectos de todos estos eventos aún no están totalmente ubicados, dimensionados y comprendidos por las ciencias naturales.
\end{abstract}

Palabras clave: Río Amazonas, Cuenca Marginal, Portal de Guayaquil, Golfo de Maracaibo.

\begin{abstract}
Evolution of the Amazon landscape from the Precambrian. This rehearsal is a study about the evolution of the Amazonian landscape from the Precambrian until the present. It is the result of an intense bibliographical research in books and national and international scientific rehearsals. It presents the geological and climatic evidences of the changes in the Amazonian, and their main implications concerning the current biodiversity; it detaches the several authors' point of view on the hypothesis of the Paleo-Amazon and the formation of his current basin. The work exposes that the geological and climatic processes went decisive for the different and multiple changes of the landscape through the time that they affected the topography, the soil, the vegetation, and the fauna of South America and of the Amazonian. There was a great impact of these events in the formation of the forest and no-forest strongholds that they contributed with the speciation and divergence of present species now in the different habitats of the Amazonian. Today, the waters of the river Amazon contribute intense sedimentation that it is transported by the tide, forming the extensive swamps of the north of Brazil. However, the effects of all these events are not still totally measured and understood for the natural sciences.
\end{abstract}

Keywords: Amazon River, Marginal Basin, Portal of Guayaquil, Gulf of Maracaibo.

INTRODUCCIÓN La Amazonia de hoy con toda su heterogeneidad de paisajes posee un pasado desconocido por el grande público. Comprenderla requiere conocimiento de su pasado y de los eventos ocurridos en este bioma. Su conformación geológica, topográfica y climática he cambiado dinámicamente desde el Precámbrico hasta el presente. Este texto aborda esta dinámica y su consecuente evolución a través del tiempo, desde Gondwana, la separación de África y Suramérica, y el cambio de rumbo del río Amazonas desde el Pacífico hacia el Atlántico, apuntando sus principales modificaciones geológico-climáticas y sus reflejos sobre la fauna y flora amazónicos.

Tanto al Norte como al Sur de la región amazónica, los antiguos escudos arquéanos, respectivamente, Guyanés y del Brasil Central, son constituidos de granito, recubiertos por arenitos. "Entre los viejos escudos se extiende la depresión amazónica que, en el Paleozoico, se encontraba cubierta por el mar, configurando un gigantesco golfo, abierto para el Pacífico" (Katzer 1903). Estando Suramérica conectada a África en esta época, se cerraba pues el golfo al este (Sioli 1983). En consecuencia, fueron dejados por este mar sedimentos de Los periodos Siluriano, Devoniano, Carbonífero, teniendo hasta 3.000 metros de espesor que recorren por bajo toda la cuenca sedimentar amazónica.

En las orillas septentrional y meridional de la media y baja Amazonia, os sedimentos marinos afloran, se sucediendo de la periferia de la cuenca para el centro en tiras de sedimentos Silurianos, Devonianos y Carboníferos. "Durante el Mesozoico hubo una regresión marina, haciendo con que el nivel del mar bajase, así la depresión Amazónica ahora era tierra emergente. Los ríos que entonces drenaban esta tierra fluían para el Pacífico - corriendo, por tanto, el Paleo-amazonas en sentido contrario al actual 
(Ab'Saber 1996)". Durante el Jurásico y comienzos del Cretáceo ocurrieron por toda parte intrusiones de diabásio (roca eruptiva intrusiva básica, de coloración negra o verde, principalmente la augita. Este tipo de roca se distingue de los basaltos por causa de la textura ofítica. Los diabásios o doleritos aparecen más comúnmente en diques y en masas intrusitas (Guerra \& Guerra 2005)), prenunciando aparentemente la separación entre América del Sur y África. En Amazonia, estas intrusiones "alcanzaron la superficie actual, principalmente en tiras de sedimentos marinos y en puntos esparcidos de los escudos Arqueozoicos (Sioli 1983)". Se percibe entonces, que el curso del río Amazonas como lo conocemos hoy, pasó por intensas transformaciones a través de los siglos.

Para conocer y comprender la dinámica de la cuenca amazónica se debe tener en cuenta "los eventos climáticos y geológicos que tuvieron lugar en el continente Suramericano a partir de su separación de África (Galvis et al. 2006)".

CUADRO HISTÓRICO-GEOLÓGICO Para el entendimiento de las condiciones actuales de la
Amazonia es imprescindible saber cómo ellas se originaron. Es necesario conocer primero la historia geológica del planeta (Tab. 1). "Según Guerra \& Cunha (2003), las Eras, Periodos y Épocas geológicas del planeta Tierra presentan las siguientes características:

a) Era Precámbrica - presenta las rocas que primero surgieron en la superficie del planeta. La distribución de tierras ocurría en dos escudos: El Arqueo-ártico, ubicado en el polo Norte; y el Afrobrasilero-indo-malgaxe (Madagascar) en la región ecuatorial; ambos separados por el Mar de Téthis.

b) Era Paleozoica - las tierras se distribuyen de la siguiente manera: Gondwana o Indo-afro-brasilero; Tierra Canadiense o Algonquiana; Tierra Sino-siberiana o Continente de Angara; Tierra Escandinava; y Tierra Tirrenídea.

c) Era Mesozoica - la distribución de las tierras era: Continente Atlántico Norte; Continente Sinosiberiano; Continente Afro-brasilero; y Continente Austro-indo-malgaxe.

d) Era Cenozoica - presentando el desarrollo de los mamíferos, aparecimiento del hombre, grandes movimientos tectónicos y última glaciación".

Tabla 1 - Escala Geológica del Tiempo.

\begin{tabular}{|c|c|c|c|c|}
\hline Eras & Períodos & Épocas & Años & Características \\
\hline \multirow{7}{*}{ Cenozoica } & \multirow{2}{*}{ Cuaternario } & Holoceno & 11.000 & Humanos modernos. Periodo interglaciar. \\
\hline & & Pleistoceno & $1,5 \mathrm{MA}^{*}$ & Surgimiento del género Homo. Edad del Hielo. \\
\hline & \multirow{5}{*}{ Terciario } & Plioceno & $12 \mathrm{MA}$ & $\begin{array}{l}\text { Primeros hombres primitivos. Unión de Norteamérica y Suramérica; } \\
\text { elevación continental, resfriamiento. }\end{array}$ \\
\hline & & Mioceno & $23 \mathrm{MA}$ & Reintegración entre Europa y África. \\
\hline & & Oligoceno & $35 \mathrm{MA}$ & Primeros monos del Nuevo Mundo. \\
\hline & & Eoceno & $55 \mathrm{MA}$ & $\begin{array}{l}\text { Primeros caballos, ballenas, murciélagos y monos. Final de la separación de } \\
\text { Australia y Antártica. }\end{array}$ \\
\hline & & Paleoceno & $70 \mathrm{MA}$ & $\begin{array}{l}\text { Mamíferos placentarios. África y Europa se separan provisioramente. Clima } \\
\text { subtropical. }\end{array}$ \\
\hline \multirow{3}{*}{ Mesozoica } & Cretáceo & & $135 \mathrm{MA}$ & $\begin{array}{l}\text { Plantas con flores; extinción de los dinosaurios. Separación de Gondwana. } \\
\text { Inicio de la elevación andina. }\end{array}$ \\
\hline & Jurásico & & $190 \mathrm{MA}$ & Aves y mamíferos; abundancia de dinosaurios. Pangea. \\
\hline & Triásico & & $230 \mathrm{MA}$ & Primeros dinosaurios. \\
\hline \multirow{6}{*}{ Paleozoica } & Pérmico & & $280 \mathrm{MA}$ & Extinción de los trilobites. \\
\hline & Carbonífero & & $350 \mathrm{MA}$ & $\begin{array}{l}\text { Florestas formadoras de carbón; coníferas; reptiles; anfibios abundantes; } \\
\text { samambaias primitivas. }\end{array}$ \\
\hline & Devónico & & $400 \mathrm{MA}$ & Primeros anfibios. \\
\hline & Silúrico & & $440 \mathrm{MA}$ & Primeras plantas y animales terrestres. \\
\hline & Ordovícico & & $500 \mathrm{MA}$ & Primeros peces. \\
\hline & Cámbrico & & $570 \mathrm{MA}$ & Primero registro de abundante vida marina, trilobites. \\
\hline \multirow{2}{*}{ Precámbrico } & Algonquiano & & $2 \mathrm{kMA}^{* *}$ & Fósiles muy raros, consistiendo de plantas acuáticas raras. \\
\hline & Arqueano & & $4,5 \mathrm{kMA}$ & Ausencia de vida. \\
\hline
\end{tabular}

Adaptado de Hickman et al. (2003), Brown \& Lamolino (1998), Pielou (1979).

* Millones de años. ** Mil millones de años. 
EVOLUCIÓN GEOLÓGICA Antes de la separación de Suramérica y África, el borde occidental de Gondwana drenaba sus aguas hacia el Pacífico. Se debe considerar que la fragmentación de Gondwana occidental en dos continentes: Suramérica y África, es el primero de los eventos geológicos importantes en la dinámica de la actual cuenca amazónica (Galvis et al. 2006). Estos autores defiendem que "la formación de las dos ramas que componen el rift del Atlántico Sur que terminó separando estos dos continentes, antes de la penetración marina, constituyó los grandes lagos sobre los diferentes bloques en subsidencia" (Fig. 1).



Figura 1 - Fragmentación de Gondwana occidental hacia el Cretáceo. a) Rama norte del rift; b) Rama sur del rift; c) Cuenca marginal aún cubierta por el mar (Galvis et al. 2006).
"La teoría de las placas tectónicas explica la unión y separación de África y América del Sur. La depresión amazónica, antes que hubiese cualquier barrera a oeste era un golfo (Leinz \& Amaral 2003)", y la presencia de sedimentos de varias edades del Paleozoico, Siluriano, Devoniano, Carbonífero y después más tarde, "restos de sedimentos marinos del Mioceno, sirven para comprobar que el mar venía de oeste hasta un cierto tiempo (Sioli 1983)".

"El desplazamiento de Suramérica a la medida que se separaba de África hizo que su borde occidental montara sobre la placa oceánica adyacente - Placa de Nazca, lo cual produjo, por compresión, el levantamiento y plegamiento de ese borde, que terminaría formando Los Andes (Leinz \& Amaral 2003)". "Con la separación de África y Suramérica, su topografía debió ser bastante plana - peneplana - con excepción del Escudo Guayanés cuyo levantamiento tuvo lugar principalmente durante el Cretáceo (Reynaud \& do Valle 1997)". En el Mioceno, Los Andes empiezan a levantarse, "pasando entonces la joven cadena de montañas a bloquear, durante el Plioceno y todo el Plistoceno, el escurrimiento del sistema fluvial amazónico para el Pacífico (Ayres 2006)”.

\section{CUENCA MARGINAL Y LAS HIPÓTESIS: PORTAL DE GUAYAQUIL O GOLFO DE} MARACAIBO "Antes de la separación entre Suramérica y África, todo el borde occidental presentaba su drenaje para el Pacífico (Caputo 1974)". Pero con el soerguimiento de Los Andes, "en la porción oriental de esta cordillera y paralela a esta, se formó una zona deprimida - la cuenca marginal con extensión del Lago de Maracaibo hacia el sur de Bolivia (Sarmiento 1992)" (Fig. 2).

El sistema de drenaje formado por los ríos Solimões y Amazonas no era continuo como

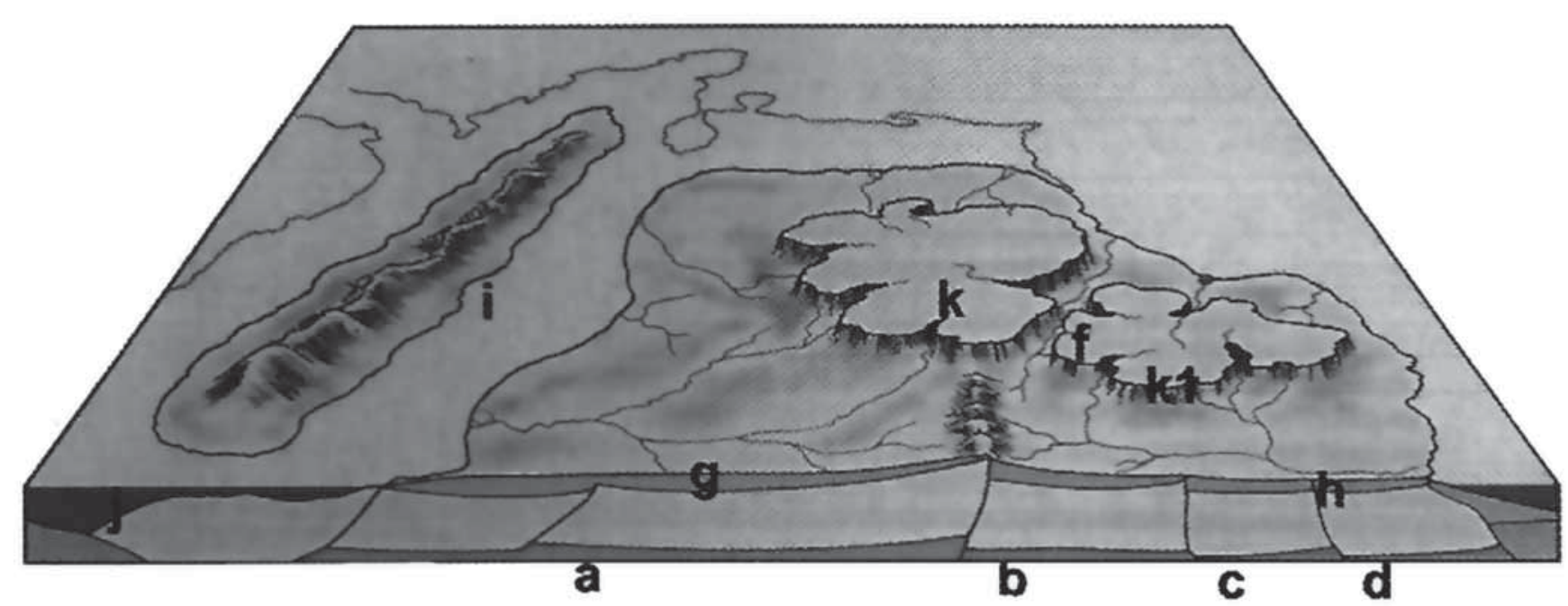

Figura 2 - Transgresión marina del Cretáceo superior. (i) Cordillera central aún está como una isla, separada del continente por la cuenca marginal. (j) Portal de Guayaquil; (a) Arco de Iquitos; (b) Arco de Purús; (c) Arco de Montealegre; (d) Arco de Gurupá; (f) Fosa de Tacutú; (g) Paleo-amazonas occidental; (h) Paleo-amazonas oriental; (k, k1) Escudo Guyanés (Galvis et al. 2006). 
actualmente, la parte occidental era separada de la oriental por una gran elevación, llamada Arco Purus (Maciente \& Ranzi 2007). "El Arco Purus actuaba como una divisoria de aguas y cortaba perpendicularmente la actual planicie amazónica, ocasionando que hacia el oriente las aguas fluyeran hacia el Atlántico - a través del Paleo-Amazonas Oriental - y hacia el occidente a la cuenca marginal (Galvis et al. 2006)" (Fig. 3).

"La apertura de esta cuenca para el Pacífico aun es materia de discusión. Hay científicos que defienden la hipótesis del Portal de Guayaquil (también conocido como Portal de Marañon) como siendo una salida para el Pacífico; y otro grupo defiende la del Golfo de Maracaibo, con salida para el Caribe (Galvis et al. 2006)". La hipótesis del Portal de Guayaquil fue propuesta por Katzel (1903), quién planteó que el PaleoAmazonas corría hacia el Pacífico y desembocaba en un gran golfo que paulatinamente se cerró. "Caputo (1974) se refiere a una drenaje de la cuenca amazónica, durante el Cretáceo, que estaba en comunicación periódica con el Pacífico". Entonces, según esta hipótesis, el PaleoAmazonas drenaba la Amazonia y corría para el Pacífico, desembocando en un golfo, que con el tiempo se cerró lentamente (Sioli 1983). Además es importante decir, que esta cuenca marginal drenaba la parte occidental del escudo Guayanés y del escudo Brasilero. "Brooks et al. (1981) también consideran la existencia de este portal y defienden que por allí los peces marinos (por ejemplo las rayas) penetraron a la cuenca marginal" (Fig. 3).

"La Falla de Romeral, que se extiende desde la desembocadura del río Magdalena siguiendo el curso medio y alto de los ríos Cauca y Guacas (en Colombia) hasta Guayaquil, fue el borde continental Pacífico durante la transición Cretáceo-Terciario, afirma Duque-Caro (1980)". "A principios del Terciario, la cuenca en su sector norte empezó a colmatarse con sedimentos provenientes del Escudo Guyanés, pero al incrementarse la actividad tectónica en Los Andes, en el Mioceno Medio, pasaron a predominar los sedimentos provenientes de la cordillera andina (Hoorn 1993)".

De otra parte, Lundberg et al. (1998), afirman que "la cuenca marginal captaba el drenaje de un área comparable a de la cuenca amazónica actual, y se extendía desde Bolivia hasta el Lago de Maracaibo (en Venezuela), desembocando en el Caribe" (Fig. 4). En el Paleoceno, "la Formación Guaduas de la cordillera oriental colombiana presenta numerosos paleo-cauces meándricos, muchos de ellos en dirección orienteoccidente procedentes del Escudo Guyanés, o en dirección contraria hacia el suroriente, en lo que hoy es el alto río Magdalena (Lundberg 1997)".

Esto nos lleva a pensar en más que un gran río continuo, y sí en una "serie de depresiones mal drenadas y en distintos grados de hundimientos, como está evidenciado por los diferentes espesores de sedimentos en cada una de ellas. Así, estas depresiones o cuencas pudieron intercomunicarse esporádicamente (Radelli 1967)". Estas depresiones son los llamados 'Lagos o Formaciones del Terciario' - como el Lago Pebas, del Mioceno, llamado Formación Solimões en Brasil). De acuerdo con "Galvis et al. (2006), estos lagos tuvieron influencia marina como lo evidencia la presencia de foraminíferos" y de "polen de mangle en el caso del Lago Pebas (Colinavaux 1996)”.

La influencia marina, argumenta "Lundberg et al. (1998), en estos lagos provenía del mar del Caribe al norte y correspondía a transgresiones marinas que penetraron a lo largo de la cuenca marginal"; ya "Hoorn (1993) defiende que esta influencia provenía de una entrada mucho más cercana y no tan alejada como el Caribe". Caso se considere lo planteado por "Lundberg et al. (1998), la cual hubo una conexión restringida entre

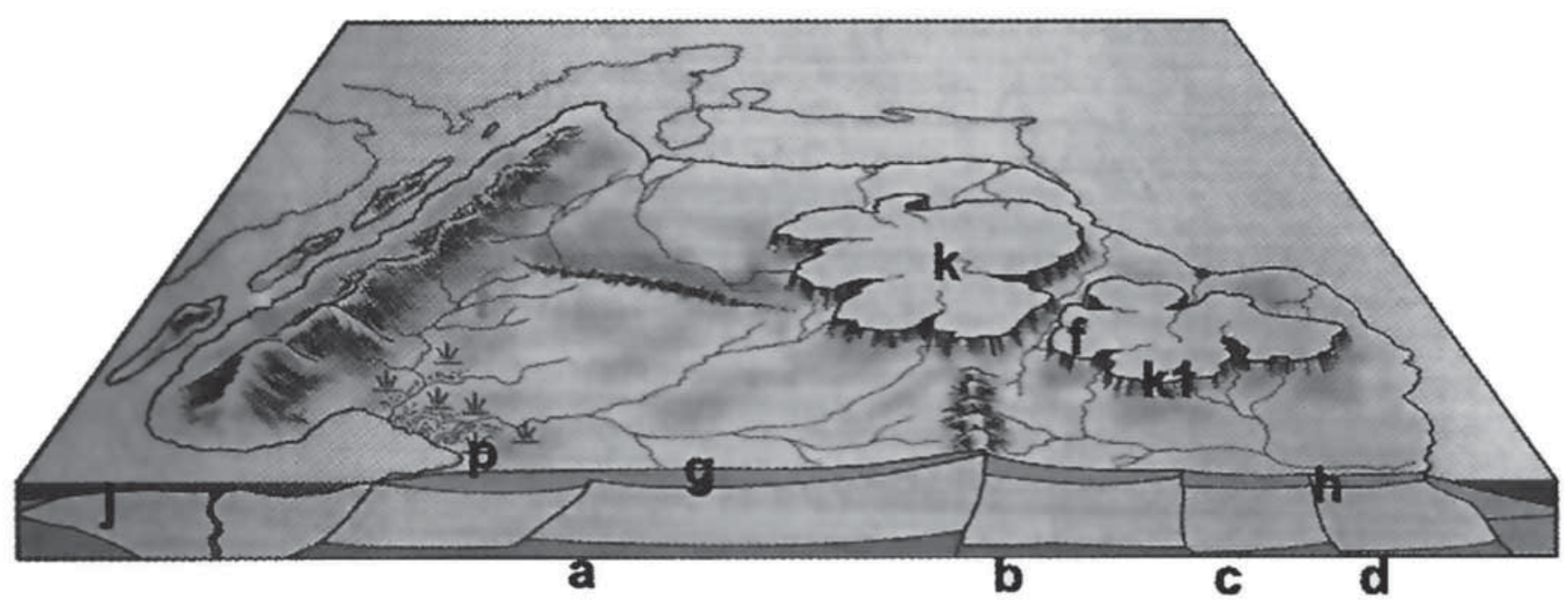

Figura 3 - Terciario medio. (j) Portal de Guayaquil aún abierto; (l) Cordillera occidental en emergencia; (a) Arco de Iquitos; (b) Arco de Purús; (c) Arco de Montealegre; (d) Arco de Gurupá; (f) Fosa de Tacutú; (g) Paleoamazonas occidental; (h) Paleo-amazonas oriental; (k, kl) Escudo Guyanés; (p) Formación Solimões (Pebas) (Galvis et al. 2006). 


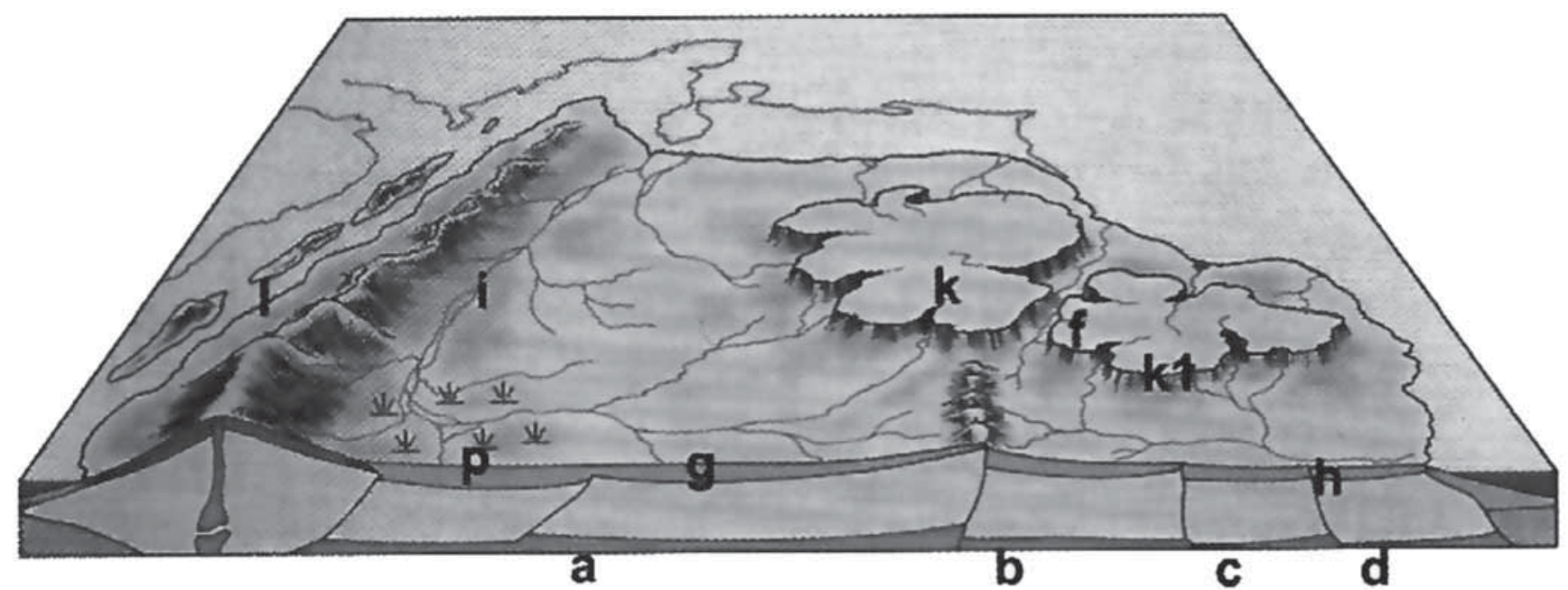

Figura 4 - Terciario medio. (i, g) Sistema Amazonas-Magdalena-Orinoco y su salida al Caribe; (a) Arco de Iquitos; (b) Arco de Purús; (c) Arco de Montealegre; (d) Arco de Gurupá; (f) Fosa de Tacutú; (h) Paleo-amazonas oriental; (k, k1) Escudo Guyanés; (p) Formación Solimões (Pebas) (Galvis et al. 2006).

el Lago Pebas y el Caribe, y que en este lago se produjo un hundimiento de algunas decenas de metros"; se debe tener en cuenta que "una transgresión marina no podría penetrar más de mil kilómetros a una cuenca de tales características bajo condiciones de precipitación similares a las actuales, pues simplemente produciría un enorme embalse de agua dulce (Hoorn 1993, Brooks et al. 1981, Galvis et al. 2006)".

La Formación Pebas se formó durante un lapso de tiempo de aproximadamente 16 millones de años, y debió comprender múltiples eventos climáticos que pudieran dar lugar a toda la gama de paisajes que se pueden presentar en una zona de subsidencia y deficientemente drenada. Dentro de estos eventos, el más improbable fue que haya ocurrido la penetración desde el Caribe de una transgresión marina. Esto es corroborado por "Frailey et al. (1988) que en su propuesta del lago amazónico Pleistoceno-Holoceno producido por basculamiento y transgresión marina, no plantean una penetración de aguas marinas a la cuenca, sino un enorme embalse de aguas dulces".

En oposición, para "Lundberg (1997), los fósiles de peces de La Venta en el desierto de Tatacoa (Alto Río Magdalena) corresponden a un periodo datado entre 20 y 11,8 millones de años, anterior a la formación del valle superior del río Magdalena. Estos fósiles hacen parte de la fauna que vivía en zonas pantanosas que bordeaban el costado occidental de dicha cuenca, así el río Amazonas-Orinoco-Magdalena formaba un único sistema que desaguaría en el Caribe". Durante el Mioceno, el levantamiento de la Cordillera Oriental empujó hacia arriba el fragmento de escudo correspondiente hoy a la Sierra de La Macarena, elevando el Arco Vaupés. Con eso, principia a formarse la divisoria de aguas entre la Amazonia y la Orinoquia, y progresivamente se fue cesando el flujo de agua de la actual Alta Amazonia, hacia el Caribe. "En ese mismo tiempo, la zona del Alto Amazonas, donde está la Formación Solimões, se colmató de sedimentos andinos para finalmente, hace 8 millones de años, terminar drenando hacia el oriente por sobre el Arco Purus, con lo que se formó el Amazonas actual (Barros et al. 1977)".

EVOLUCIÓNCLIMÁTICA Desde la separación de Suramérica y África, la tensión entre las placas Nazca y Suramericana empezó a formar Los Andes. "De inicio sus alturas no eran considerables que pudieran incidir sobre la condensación y lluvias hasta el Mioceno (Marshall \& Sempere 1993)". Para Reynaud \& do Valle (1997), "el Estado de Roraima ubicado en el extremo norte de la cuenca amazónica brasilera, durante el Terciario Inferior se caracterizó por un clima árido y semiárido". Esto se debe que a nivel mundial, "en el transcurso del Terciario, se presentó una disminución gradual de la temperatura media y por consiguiente de las precipitaciones, que culminó con las glaciaciones del Cuaternario (Haffer \& Prance 2002)". Esto implicó en una paulatina "acumulación de hielo en los casquetes polares, y el ulterior descenso del nivel del mar - retracción marina (Ab'Saber 1982)".

Los escasos indicios referentes al clima del Terciario han sido aportados por los paleo-botánicos que estudiaron la evolución de plantas angiospermas (Romero 1993). Con eso, se puede considerar que en el Paleoceno existieron dos provincias: a) la Provincia de las Palmas, con flora tropical al norte de Suramérica; b) Provincia Mixta localizada hacia el sur, con un clima subtropical. Posteriormente, "en el Eoceno, la flora de la Provincia de las Palmas se diversificaría, dando origen a los dominios del Caribe, Amazónico, y Guayanés (Fig. 5).

La Provincia Mixta, en el Oligoceno, contendría diversos grupos que hoy pertenecerían a las sabanas 


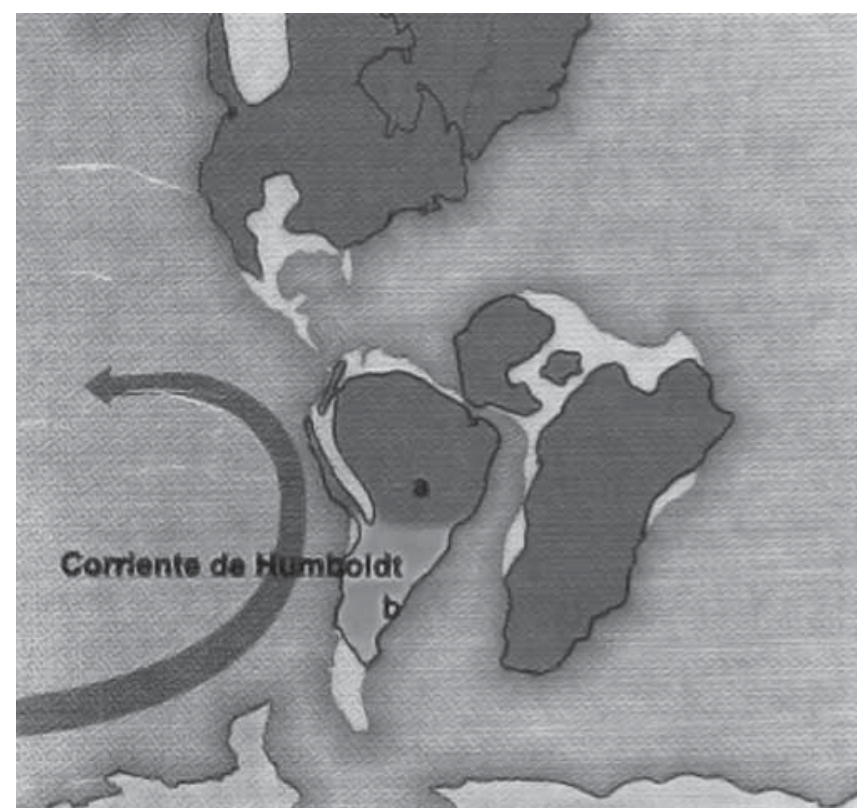

Figura 5 - En el Paleoceno, Suramérica consistía en dos provincias: (a) Provincia de las Palmas - Tropical; (b) Provincia Mixta-Subtropical (Galvis et al. 2006).

arboladas del dominio Chaco y a elementos de las zonas más áridas (Romero 1993)". En este mismo periodo, se empiezan a diversificarse la vegetación árida del Chaco y región Andino-Patagónica, y la provincia de notofagilitas del sur de Chile. Esto significa que el clima empezaba un periodo de sectorización en franjas latitudinales, surgiendo un clima más seco hacia el Trópico de Capricornio y más frío en el extremo sur (Romero 1993)" (Fig. 6).

Conjuntamente al enfriamiento gradual del Oligoceno medio, al separarse completamente Suramérica de la Antártica, "se formó una circulación marina circun-antártica de agua más fría que bajó la temperatura de la Corriente de Humboldt, y empezó a tener efectos de resurgencia sobre la plataforma continental y de inversión térmica con la consecuente desertificación de la costa adyacente (Galvis et al. 2006). Como Los Andes aún no poseían una gran elevación que pudiera impedir el proceso de desertificación, este se amplió hacia el interior del continente. "La parte sur de la cuenca marginal fue afectada por este proceso de desertificación, con la extensión de la vegetación árida del Chaco (Romero 1993)". O sea, "la cuenca marginal del Paleo-Amazonas, a partir del Oligoceno medio tendría sus cabeceras en una extensa zona de clima seco (Hooghiemstra \& Hammen 1998)".

Con respecto a los 'lagos o formaciones', "Galvis et al. (2006) apuntan que debido a las sequías, ellos eran deltas internos". Para "Lee (1990), el Lago Pebas no era una región abierta y sí una zona muy amplia en subsidencia cubierta por canales fluviales y áreas pantanosas similares a un estuario, posiblemente con un clima característico de sabanas". Todo ello lleva a creer que el paisaje preponderante en la región tropical y subtropical de Suramérica durante parte del Terciario fue de sabanas, común en superficies peneplanas y de baja elevación. Además que, según "Haffer y Prance (2002) y Ab'Saber (2002), fue muy común en el sentido oriente-occidente los reductos de selva en los flancos orientales de los accidentes geográficos de mayor altura, capaces de producir condensación y lluvia".

El efecto de condensación derivado por el levantamiento de Los Andes y de los escudos Brasilero y Guyanés hacia el final del Terciario fue contrarrestado por el enfriamiento progresivo que presidió la primera glaciación del Cuaternario. De esa manera, "Hooghiemstra \& Hammen (1998) consideran que las extensiones selváticas análogas a las actuales exclusivamente debieron presentarse en los periodos interglaciares cálidos y húmedos, cuando la cordillera andina presentaba un flanco oriental de gran altura, capaz de condensar la humedad marina transportada por los alisios del norte y del sur". Algo similar igualmente sucedió con los escudos de Brasil y Guayana, cuyos flancos orientales elevados presentaban mayores precipitaciones, siendo cubiertos de selvas húmedas.

"Por disminución general de la humedad atmosférica al bajar la temperatura durante los periodos glaciares del Cuaternario se provocó la contracción de áreas selváticas y expansión de sabanas y desiertos en las regiones tropicales (Tricart 1974)". Es posible demostrar trazos de este periodo a través de la formación de dunas en las sabanas orinocenses de Casanare, Arauca y Apure correspondientes al último período glaciar. Los periodos secos durante

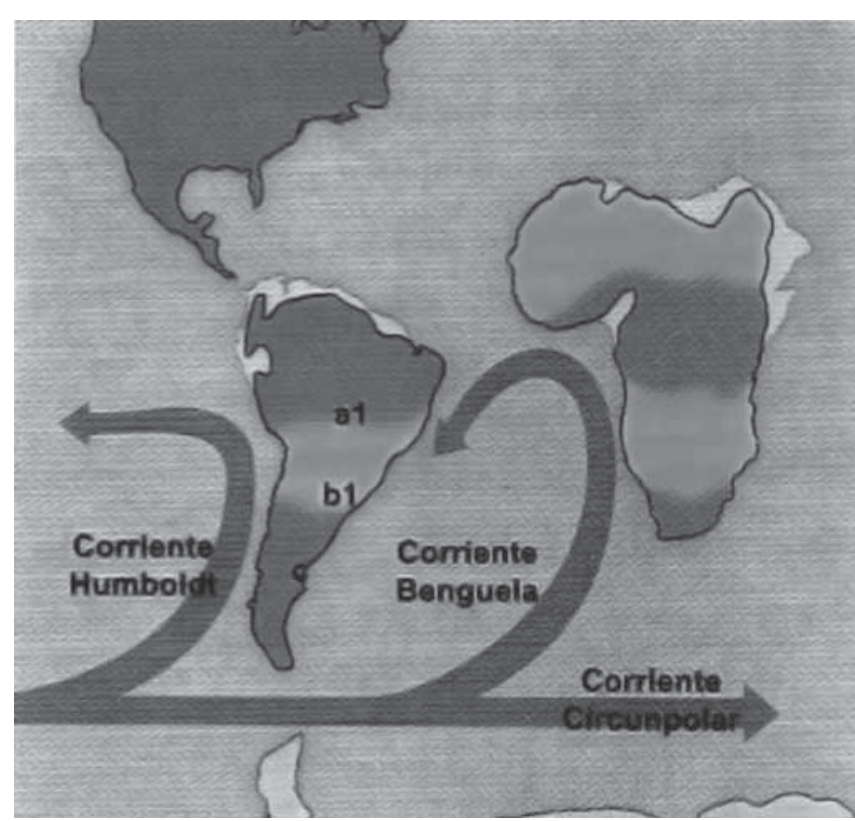

Figura 6 - A final del Oligoceno, con la formación de la corriente circumpolar y el enfriamiento de la Corriente de Humboldt, Suramérica se fraccionó en tres zonas latitudinales: (a1) Provincia Tropical; (b1) Provincia del Chaco; (c) Provincia de Notofagilitas (Galvis et al. 2006). 
las glaciaciones incidieron en la cuenca amazónica acarreando la contracción de la red fluvial. "En los valles interandinos, el efecto del fenómeno de Fönh (Sombra de Lluvia) en los periodos glaciares provocó sequías tan intensas capaces de causar la extinción de parte de la fauna íctica (Lundberg 1997)".

En algunos sitios, donde la sequía fue más aguda, la floresta húmeda fue suplantada por floresta abierta de palmeras, por sabanas y también por caatingas (tipo de vegetación característica del nordeste brasilero, formado por pequeñas árboles, comúnmente espinosas, que pierden las hojas en el curso de la larga estación seca. Entre ellas ocurren numerosas plantas suculentas, principalmente los cactáceos), antes que su ciclo retornase a floresta húmeda. Estos ciclos vegetacionales paleo-climáticos fueron completos en aquellas regiones donde la floresta fue sustituida por una vegetación no-forestal durante la fase seca. Así, la floresta y la vegetación no-forestal son antiguos en las áreas de su ocurrencia continua; y por otro lado, las florestas y sabanas ofrecieron condiciones ecológicas estables y uniformes a la fauna. La floresta húmeda fue fragmentada en bloques aislados que después se unieron bajo variadas condiciones climáticas. "Las florestas húmedas y la vegetación no-forestal, formadas durante los picos seco (frío) y húmedo (cálido) de las fases climáticas, llevaron a la reposición por pulsaciones en la evolución de la biodiversidad durante el Cuaternario y Terciario (Vrba 1992, 1993)".

\section{ZONA COSTERA ATLÁNTICA}

"En el

Cuaternario, entre 23 y 13 mil años hasta el presente (AP), el nivel de los mares descendió aproximadamente 100 metros. En este periodo de tiempo, el clima de Amazonía comportaba precipitaciones menores que las actuales y una estacionalidad pronunciada, mientras tanto el río era menos caudaloso (Ab'Saber 2002)". De esa manera, el río Amazonas se extendía hacia la línea costera rebajada y reculada. Debido al cambio del nivel de la base y a la fuerte actuación de la erosión regresiva se formaron cañones en la plataforma continental, seccionando los terrazos elaborados en el Terciario.

Cuando el mar subió, "a partir de 12.000 AP, alcanzando un nivel aproximado de 3 metros arriba del actual, provocó el ahogamiento a lo largo de la desembocadura norte y sur del río Amazonas alrededor de la isla Marajó. Durante su ascenso, el mar en frente de las tierras altas de la isla ejecutó un proceso abrasivo profundo. Hasta aquél momento no existían las planicies costeras que surgen en el norte y nordeste de la costa brasilera (Ab'Saber 2002)". En un sube y baja, el mar retrocedió de su línea de costa Paleo-marajoara, empezando los procesos de intensa sedimentación formadores de los actuales campos inundables de Marajó.

"Por ocasión del optimum climático, el nivel del mar logró su nivel máximo (3 metros más que el actual) entre 6 y 5 mil AP. El paisaje local de esta época presentaba lagos semi-marinos, ya que las aguas marinas con salinidad disminuida, envolvieron la porción occidental de Marajó (Ayres 2006)", haciendo "recular la condición del estuario hacia el bajo río Tocantins y bajo río Amazonas (Ab'Saber 1982)". Así, "la porción oriental de Marajó estaba sometida a una ingresión rasa, de fuerte abrasión, preparando la plataforma baja que vendría a recibir la sedimentación más reciente que caracteriza los campos inundables de Marajó (Ab’Saber 1996)”. Hoy, el río Amazonas tributa una extraordinaria cantidad de sedimentos en la zona del estuario. Estos sedimentos son el principal formador del suporte geológico y ecológico de la costa norte de Brasil. Una parte importante de estos sedimentos es restituida para las orillas del estuario y márgenes terminales de algunos ríos de mayor porte.

CONCLUSIONES La Amazonia como es percibida hoy, presenta características muy distintas de los tiempos pretéritos. Los mega-eventos geológicos y climáticos, en grande o pequeña escala, afectaron la región. La transformación de Gondwana en dos continentes hizo con que una red hidrográfica y grandes comunidades de fauna y flora ya constituidas fuesen separadas, y una nueva reacomodación y readaptación tuvieron que empezar en un nuevo y diferente paisaje.

La apertura del Paleo-Amazonas para el Pacífico (o para el Caribe) y su posterior cierre fue factor determinante para la mezcla de la comunidad íctica del Amazonas, Magdalena y Orinoco, mismo que hoy no haga representaciones expresivas comunes a las tres cuencas. Actualmente, el río Amazonas deposita enormes cantidades de sedimentos alrededor de Marajó, que permanecen disponibles a la acción de las mareas, y de ahí se originan las grandes áreas de manglares de los estados de Amapá y Pará.

Los cambios climáticos por el levantamiento de Los Andes y por las glaciaciones del Terciario y Cuaternario (con sus largos períodos de sequías) ejercieron, no solamente, importantes alteraciones en el suelo y en la hidrografía amazónica, pero también establecieron los tipos y la distribución de la vegetación suramericana en general, y de la amazónica, en particular.

Estos diferentes tipos de vegetación fueron importantes actores para la dinámica de la especiación, así como sirvieron como multiplicadores y mantenedores, hasta hoy, de endemismos y de una diversidad muy amplia de formaciones vegetales adaptadas a condiciones que as veces se presentan bajo alto estrese ambiental.

Agradecimientos Agradezco al señor Mário Júnior Polônia Acampa, Técnico de Informática del Centro de Estudios Superiores de Tabatinga (Universidad del Estado del Amazonas) por el tratamiento de las ilustraciones. A Pablo Alberto Palacios Hernández, mi tutor en la maestría y profesor de la disciplina Ecología y Geografía de la Amazonia. Y a todos mis alumnos de Geología y Ecología de la UEA-Tabatinga, que motivaron la investigación y ejecución de este trabajo. 


\section{Referencias}

Ab'Saber A.N. 2002. Bases para o estudo dos ecossistemas da Amazônia brasileira. Estudos Avançados, 16(45):7-30.

Ab'Saber A.N. 1996. Ecossistemas Continentais. In: Kacowicz Z. \& Oliveira E.M. (coords.) Relatório da qualidade do meio ambiente. Brasília, SEMA, p. 312.

Ab'Saber A.N. 1982. The paleoclimate and paleoecology of Brazilian Amazônia. In: Prance G.T. (ed.) Biological Diversification in the Tropics. New York, Columbia University Press, p. 41-59.

Ayres J.M. 2006. As Matas de Várzea do Mamirauá: Médio Solimões. Belém, Sociedade Civil Mamirauá, 123 p.

Barros et al. 1977. Projeto RADAMBRASIL. Folhas SB/ SC. 18 Javari/Contamana. Rio de Janeiro, DNPM, Levantamento de Recursos naturais, Volume 13, 438 p.

Brooks D., Torzón T., Mayers M. 1981. Freshwater stingrays (Potamotrygonidae) and their helminth parasites: testing hypotheses of evolution and coevolution. In: Funk A. \& Brooks D. (eds.) Advances in Cladistics. New York, New York Botanical Garden Press, p. 147-175.

Brown J.H. \& Lamolino M.V. 1998. Biogeography. Sunderland, Sinauer Assoc., 391 p.

Caputo M.V. 1974. Interpretação ambiental do sistema cretáceo nas bacias do Acre, Ucayali e Pastaza. Belém, PETROBRÁS-RENOR, Relatório Técnico 680-A, 406 p.

Colinvaux P.A. 1996. Quaternary environmental history and forest diversity in the Neotropics. In: Jackson J.B.C., Budd A.F., Coates A.G. (eds.) Evolution and environment in tropical America. Chicago, University Chicago Press, p. 359-405.

Duque-Caro H. 1980. Geotectonica y evolucion de la region noroccidental colombiana. Boletín Geológico INGEOMINAS, 23(3):4-37.

Frailey C.D., Lavínia E.L., Rancy A., Pereira de Souza J. 1988. A proposed Pleistocene/Holocene lake in the Amazon Basin and its significance to Amazonian geology and biogeography. Acta Amazonica, 18:119-143.

Galvis G., Mojica I., Duque S.R., Castellanos C., SánchesDuarte P., Arce M., Gutiérrez A., Jiménez L.F., Santos M., Vejarano-Rivadeneira S., Arbeláez F., Prieto E., Leiva M. 2006. Peces del medio Amazonas. Región de Leticia. Bogotá, Conservación Internacional, 547 p.

Guerra A.T. \& Guerra A.J.T. 2005. Novo dicionário geológicogeomorfológico. Rio de Janeiro, Bertrand Brasil, 648 p.

Guerra A.J.T. \& Cunha S.B. 2003. Geomorfologia do Brasil. Rio de Janeiro, Bertrand Brasil, 388 p.

Haffer J. \& Prance G.T. 2002. Impulsos climáticos da evolução na Amazônia durante o Cenozóico: sobre a teoria dos Refúgios da diferenciação biótica. Estudos Avançados, 16(46):175- 219.

Hickman C.P., Roberts L.S., Larson A. 2003. Princípios Integrados de Zoologia. Rio de Janeiro, GuanabaraKoogan, 846 p.

Hooghiemstra H. \& Hammen T.V. 1998. Neogene and Quarternary development of the neotropical rain forest: the forest refugia hypothesis, and literature overview. Earth-Science Reviews, 44:147-183.

Hoorn C. 1993. Marine incursions and the influence of Andean tectonics on the Miocene depositional history of northwestern Amazônia: results of a palynostratigraphics study. Paleog., Paleoclim., Paleoecol., 105:267-309.

Katzer F. 1903. Grundüge des Geologie des unteren Amazonas-Gebietes des States Pará in Brasilien. Leipzig, Verlag von Max Weg, 298 p.

Lee D. 1990. Okavango Delta, Old Africa's Last Refuge. National Geographic, 178(6):39-69.

Leinz V. \& Amaral S.E. 2003. Geologia Geral. São Paulo, Companhia Editora Nacional, 399 p.

Lundberg J. 1997. Fishes of the La Venta fauna: additional taxa biotic and paleoenvironmental implications. In: Kay R., Madden R., Cifelli R. \& Flynn J. (eds.) Vertebrate paleontology in the Neotropics: the Miocene fauna of La Venta Colombia. Washington, Smithsonian Institution Scholarly Press, p. 67-91.

Lundberg J., Marshall L., Guerrero J., Horton B., Malabarba M., Wesseling F. 1998. The stage for Neotropical fish diversification: a history of Tropical South American Rivers. In: Malabarba L.R.E., Reis R., Vari Z.M., Lucena C.A. (eds.) Phylogeny and classification of Neotropical fishes. Porto Alegre, Edipucrs, p. 213-230.

Maciente A.R. \& Ranzi A. 2007. Megafauna do Pleistoceno. Rio Branco, Fundação Elias Mansour, 9 p.

Marshall L. \& Sempere T. 1993. Evolution of the Neotropical Cenozic land Mamal Fauna an its geochronologic, stratigraphic and tectonic context. In: Goldblatt P. (ed.) Biological Relationship between Africa and South America. New Haven, Yale University Press, p. 329-392.

Pielou E.C. 1979. Biogography. New Cork, Wiley, 562 p.

Radelli L. 1967. Geologie des Andes Colombienes. Travaux du laboratoire de Geologie de la Faculté des Sciences de Grenoble - Memories, 6:11-469.

Reynaud S.C. \& do Vale J.F.Jr. 1997. Mudanças climáticas e evolução da paisagem em Roraima: uma resenha do Cretáceo ao Recente. In: Imbrózio R., Gondim E.J., Castellon E.G. (eds.) Homem, ambiente e ecologia no estado de Roraima. Manaus, INPA, p. 45-64.

Romero E. 1993. South American Paleofloras. In: Goldblatt P. (ed.) Biological Relationship between Africa and South America. New Haven, Yale University Press, p. 62-85.

Sarmiento R. 1992. Palinología de la Formación Guaduas - Estratigrafía y sistemática. Boletín Geológico INGEOMINAS, 32(1-3):45-126.

Sioli H. 1983. Fundamentos da ecologia da maior região de florestas tropicais. Petrópolis, Vozes, 72 p.

Tricart J. 1974. Existence de periodes seches au Quaternaire em Amazonie et dans des regions voisines. Rev. Géomorph. Dynam., 4:145-158.

Vrba E.S. 1992. Mammals as a key to evolutionary theory. Journal of Mammal, 73:1-28.

Vrba E.S. 1993. Mammal evolution in the African Neogene and a new look at the Great American Interchange. In: P. Goldblatt (ed.) Biological relationships between Africa and South America. New Have, Yale University Press, p. 393-432.

Manuscrito ID 14690 Submetido em 29 de junho de 2009 Aceito em 21 de dezembro de 2011 